\title{
Stability and Uniform Boundedness in Multidelay Functional Differential Equations of Third Order
}

\author{
Cemil Tunç and Melek Gözen \\ Department of Mathematics, Faculty of Sciences, Yüzüncü Yıl University, 65080 Van, Turkey \\ Correspondence should be addressed to Cemil Tunç; cemtunc@yahoo.com
}

Received 11 February 2013; Accepted 18 April 2013

Academic Editor: Tonghua Zhang

Copyright (C) 2013 C. Tunç and M. Gözen. This is an open access article distributed under the Creative Commons Attribution License, which permits unrestricted use, distribution, and reproduction in any medium, provided the original work is properly cited.

We consider a nonautonomous functional differential equation of the third order with multiple deviating arguments. Using the Lyapunov-Krasovskiì functional approach, we give certain sufficient conditions to guarantee the asymptotic stability and uniform boundedness of the solutions.

\section{Introduction}

Differential equations of third order are valuable tools in the modeling of many phenomena in various fields of science and engineering (Chlouverakis and Sprott [1], Cronin-Scanlon [2], Eichhorn et al. [3], Friedrichs [4], Linz [5], and Rauch [6]). In reality, the stability and boundedness of solutions of certain nonlinear differential equations of the third order have been received intensive attentions by authors (Ademola et al. [7], Afuwape and Castellanos [8], Chukwu [9], Ezeilo $([10,11])$, Hara [12], Mehri and Shadman [13], Ogundare and Okecha [14], Omeike [15], Reissig et al. [16], Swick [17], Tejumola ([18, 19]), Tunç [20-33], and Yoshizawa [34]).

In 2009, Omeike [15] considered the nonlinear differential equation of the third order with the constant delay $r(>0)$ :

$$
\dddot{x}+a(t) \ddot{x}+b(t) g(\dot{x})+c(t) h(x(t-r))=p(t),
$$

and he discussed the stability and boundedness of solutions of this equation.

In this paper, instead of the above equation, we consider the nonautonomous differential equation of the third order with multiple deviating arguments:

$$
\dddot{x}+a(t) \ddot{x}+n b(t) g(\dot{x})+c(t) \sum_{i=1}^{k} h_{i}\left(x\left(t-r_{i}\right)\right)=p(t) \text {, }
$$

where $r_{i}$ are certain positive constants, and $a(t), b(t), c(t)$, $g(\dot{x}), h(x)$ and $p(t)$ are real valued and continuous functions in their respective arguments with $g(0)=h(0)=0, k=n$. The existence and uniqueness of the solutions of (2) are also assumed.

The motivation for this paper is a result of the researches mentioned regarding ordinary differential equations of the third order. It follows that the equation discussed in [15] is a special case of (2). Our aim is to improve the results established in [15] from one deviating argument to the multiple deviating arguments for the asymptotic stability and uniform boundedness of solutions. This work contributes to and complements previously known results on the topic in the literature, and it may be useful for researchers working on the qualitative behaviors of solutions. It should be noted that in recent years scores of papers have been published on the qualitative behaviors of solutions (stability of solutions, boundedness of the solutions, existence of the periodic solutions, etc.) of the functional differential equations of the second order with multiple deviating arguments. However, very little attention was given to stability and boundedness of functional differential equations of the third order with multiple deviating arguments ([32]). Therefore, it is worth investigating the qualitative behaviors of solutions in multidelay functional differential equations of the third order. This case is the novelty of the present paper. It should also be noted 
that the results to be established here are different from those in Tunç [20-33] and the literature.

\section{Main Results}

Let $p(t) \equiv 0$.

Theorem 1. One assumes that there exist positive constants $a, b, c, \rho_{i}, \alpha, \mu_{i}, \delta_{1}, \delta_{2}, \delta_{5}, \delta_{6}$, and $L$ such that the following conditions hold:

(i) $h(0)=g(0)=0, h_{i}(x) / x \geq \mu_{i}, x \neq 0$,

$h_{i}^{\prime}(x) \leq \rho_{i}, g(y) / y \geq b>0, \quad y \neq 0(i=1,2, \ldots, k)$,

(ii) $0<\delta_{1} \leq c(t) \leq b(t),-L \leq b^{\prime}(t) \leq c^{\prime}(t) \leq 0,0<a<$ $a(t)$.

If

$$
\begin{gathered}
\frac{b}{\rho_{i}}>\alpha>\frac{1}{a}, \\
\frac{1}{2} a^{\prime}(t) \leq \delta_{2}<\delta_{1}\left(n b-\alpha \sum_{i=1}^{k} \rho_{i}\right), \\
\sum_{i=1}^{k} r_{i}<\min \left\{\frac{2 \delta_{5}}{L c(\alpha+2)}, \frac{\delta_{6}}{L c \alpha}\right\},
\end{gathered}
$$

then every solution $x \equiv x(t)$ of (2) is uniform bounded and satisfies

$$
x(t) \longrightarrow 0, \quad \dot{x}(t) \longrightarrow 0, \quad \ddot{x}(t) \longrightarrow 0 \quad \text { as } t \longrightarrow \infty .
$$

Remark 2. It should be noted that it follows from (ii) that $b(t)$ and $c(t)$ are nonincreasing functions on $[0, \infty)$. Therefore, since these functions are continuous on this interval and bounded below by $\delta_{1}>0$, they are bounded on $[0, \infty)$ and the limit of each exists as $t \rightarrow \infty$. Since $L$ in (ii) is an arbitrary selected bound, we can also assume the following estimates:

$$
\begin{gathered}
0<\delta_{1} \leq c(t) \leq b(t) \leq L, \\
\lim _{t \rightarrow \infty} c(t)=c_{0}, \quad \lim _{t \rightarrow \infty} b(t)=b_{0}, \\
\delta_{1} \leq c_{0} \leq b_{0} \leq L .
\end{gathered}
$$

Proof. We write (2) in the system form as follows:

$$
\begin{gathered}
\dot{x}=y, \\
\dot{y}=z, \\
\dot{z}=-a(t) z-n b(t) g(y)-c(t) \sum_{i=1}^{k} h_{i}(x) \\
+c(t) \sum_{i=1}^{k} \int_{t-r_{i}}^{t} h_{i}^{\prime}(x(s)) y(s) d s .
\end{gathered}
$$

Define a Lyapunov-Krasovskiì functional ([35]) $V(\cdot)=$ $V\left(t, x_{t}, y_{t}, z_{t}\right)$ by

$$
\begin{aligned}
V(\cdot)= & c(t) H(x)+n \alpha b(t) G(y)+\alpha c(t) y \sum_{i=1}^{k} h_{i}(x) \\
& +\frac{1}{2} a(t) y^{2}+\frac{1}{2} \alpha z^{2}+y z \\
& +\sum_{i=1}^{k} \lambda_{i} \int_{-r_{i}}^{0} \int_{t+s}^{t} y^{2}(\theta) d \theta d s
\end{aligned}
$$

where $H(x) \equiv \int_{0}^{x} \sum_{i=1}^{k} h_{i}(s) d s, G(y) \equiv \int_{0}^{y} g(\xi) d \xi$, and $\lambda_{i}$ are certain positive constants, which will be determined later in the proof.

This functional can be arranged as follows:

$$
V(\cdot)=V_{1}+\frac{1}{2} V_{2}+\sum_{i=1}^{k} \lambda_{i} \int_{-r_{i}}^{0} \int_{t+s}^{t} y^{2}(\theta) d \theta d s
$$

where

$$
\begin{gathered}
V_{1}=c(t)\left[H(x)+n \alpha \frac{b(t)}{c(t)} G(y)+\alpha y \sum_{i=1}^{k} h_{i}(x)\right], \\
V_{2}=a(t) y^{2}+\alpha z^{2}+2 y z .
\end{gathered}
$$

Using the assumptions of Theorem 1, it follows that $\alpha a(t) \geq \alpha a>1$ since $\alpha>a^{-1}$ and $\alpha a(t)-1>0$.

Thus, there exist constants $\delta_{1}>0$ and $\delta_{3}>0$ such that

$$
\begin{aligned}
V_{2} & =a(t) y^{2}+\alpha z^{2}+2 y z \\
& =a(t)\left[y+\frac{z}{2 a(t)}\right]^{2}+\frac{1}{4 a(t)}[4 \alpha a(t)-1] z^{2} \\
& \geq \frac{1}{2} \delta_{3} y^{2}+\frac{1}{2} \delta_{3} z^{2} \\
V_{1} & \geq \delta_{1}\left[H(x)+\frac{1}{2} n \alpha b y^{2}+\alpha y \sum_{i=1}^{k} h_{i}(x)\right]
\end{aligned}
$$

since $b(t) / c(t) \geq 1, c(t) \geq \delta_{1}>0$, and $g(y) / y \geq$ $b>0$ imply that $G(y) \geq(1 / 2) b y^{2}$. Further, using the assumptions of Theorem 1 and $k=n$, it follows that 


$$
\begin{aligned}
H(x)+ & \frac{1}{2} n \alpha b y^{2}+\alpha y \sum_{i=1}^{k} h_{i}(x) \\
= & \frac{1}{2}\left[2 H(x)+n \alpha b y^{2}+2 \alpha y \sum_{i=1}^{k} h_{i}(x)\right] \\
= & \frac{1}{2}\left\{\frac { \alpha } { b } \left[\left(b y+h_{1}(x)\right)^{2}+\left(b y+h_{2}(x)\right)^{2}\right.\right. \\
& \left.+\cdots+\left(b y+h_{n}(x)\right)^{2}\right] \\
\geq & \frac{1}{2} \frac{\alpha}{b}\left\{\left(b y+h_{1}(x)\right)^{2}+\left(b y+h_{2}(x)\right)^{2}\right. \\
\geq & \sum_{i=1}^{k} \int_{0}^{x}\left(1-\frac{\alpha \rho_{i}}{b}\right) \mu_{i} s d s \\
\geq & \sum_{i=1}^{k} \int_{0}^{x}\left(1-\frac{\alpha \rho_{i}}{b}\right) \frac{h_{i}(s)}{s} s d s \\
+\sum_{i=1}^{2} \int_{0}^{x}\left(1-\frac{\alpha \rho_{i}}{b}\right) \mu_{i} x^{2}=\frac{\delta_{4}}{2} x^{2} & \left.\frac{\alpha h_{i}^{\prime}(s)}{b}\right) h_{i}(s) d s
\end{aligned}
$$

so that

$$
V_{1} \geq \frac{\delta_{1} \delta_{4}}{2} x^{2}
$$

where

$$
\delta_{4}=\sum_{i=1}^{k}\left(1-\frac{\alpha \rho_{i}}{b}\right) \mu_{i}
$$

In view of the previous discussion, we can get

$$
\begin{aligned}
V(\cdot) \geq & \left(\frac{\delta_{1} \delta_{4}}{2}\right) x^{2}+\frac{\delta_{3}}{4} y^{2}+\frac{\delta_{3}}{4} z^{2} \\
& +\sum_{i=1}^{k} \lambda_{i} \int_{-r_{i}}^{0} \int_{t+s}^{t} y^{2}(\theta) d \theta d s .
\end{aligned}
$$

Using a basic calculation, the time derivative of $V(\cdot)$ along solutions of (6) results in

$$
\begin{aligned}
& \frac{d}{d t} V(\cdot)=c^{\prime}(t) H(x)+c(t) H^{\prime}(x)+n \alpha b^{\prime}(t) G(y) \\
& +n \alpha b(t) G^{\prime}(y) \\
& +\alpha c^{\prime}(t) y \sum_{i=1}^{k} h_{i}(x)+\alpha c(t) y^{\prime} \sum_{i=1}^{k} h_{i} \\
& +\alpha c(t) y x^{\prime} \sum_{i=1}^{k} h_{i}^{\prime}(x) \\
& +\frac{1}{2} a^{\prime}(t) y^{2}+a(t) y y^{\prime}+\alpha z z^{\prime}+y^{\prime} z+y z^{\prime} \\
& +\sum_{i=1}^{k} \lambda_{i} \int_{-r_{i}}^{0}\left[y^{2}(t)-y^{2}(t+s)\right] d s \\
& =c^{\prime}(t) H(x)+c(t) y \sum_{i=1}^{k} h_{i}(x)+n \alpha b^{\prime}(t) G(y) \\
& +n \alpha b(t) z g(y) \\
& +\alpha c^{\prime}(t) y \sum_{i=1}^{k} h_{i}(x)+\alpha c(t) z \sum_{i=1}^{k} h_{i}(x) \\
& +\alpha c(t) y^{2} \sum_{i=1}^{k} h_{i}^{\prime}(x) \\
& +\frac{1}{2} a^{\prime}(t) y^{2}+a(t) y z+\alpha z \\
& \times\left[-a(t) z-n b(t) g(y)-c(t) \sum_{i=1}^{k} h_{i}(x(t))\right. \\
& \left.+c(t) \sum_{i=1}^{k} \int_{t-r_{i}}^{t} h_{i}^{\prime}(x(s)) y(s) d s\right] \\
& +z^{2}+y[-a(t) z-n b(t) g(y) \\
& -c(t) \sum_{i=1}^{k} h_{i}(x(t)) \\
& \left.+c(t) \sum_{i=1}^{k} \int_{t-r_{i}}^{t} h_{i}^{\prime}(x(s)) y(s) d s\right] \\
& +\sum_{i=1}^{k} \lambda_{i} r_{i} y^{2}-\sum_{i=1}^{k} \lambda_{i} \int_{t-r_{i}}^{t} y^{2}(s) d s
\end{aligned}
$$




$$
\begin{aligned}
= & c^{\prime}(t) H(x)+\alpha c^{\prime}(t) y \sum_{i=1}^{k} h_{i}(x)+n \alpha b^{\prime}(t) G(y) \\
& +\frac{1}{2} a^{\prime}(t) y^{2} \\
& -\left[n y b(t) g(y)-\alpha c(t) y^{2} \sum_{i=1}^{k} h_{i}^{\prime}(x)-\sum_{i=1}^{k} \lambda_{i} r_{i} y^{2}\right] \\
& -[\alpha a(t)-1] z^{2}+\alpha z c(t) \sum_{i=1}^{k} \int_{t-r_{i}}^{t} h_{i}^{\prime}(x(s)) y(s) d s \\
& +y c(t) \sum_{i=1}^{k} \int_{t-r_{i}}^{t} h_{i}^{\prime}(x(s)) y(s) d s \\
& -\sum_{i=1}^{k} \lambda_{i} \int_{t-r_{i}}^{t} y^{2}(s) d s .
\end{aligned}
$$

Using $h_{i}^{\prime}(x) \leq \rho_{i}, c(t) \leq L$, and the estimate $2|e f| \leq e^{2}+$ $f^{2}$, we have

$$
\begin{aligned}
& \alpha z c(t) \sum_{i=1}^{k} \int_{t-r_{i}}^{t} h_{i}^{\prime}(x(s)) y(s) d s \\
& \leq \alpha c(t)|z| \sum_{i=1}^{k} \int_{t-r_{i}}^{t} c|y(s)| d s \\
& \leq \frac{1}{2} \alpha c(t) z^{2} \sum_{i=1}^{k} r_{i} \rho_{i}+\frac{1}{2} \alpha c(t) \sum_{i=1}^{k} \int_{t-r_{i}}^{t} \rho_{i} y^{2}(s) d s \\
& \leq \frac{1}{2} L \alpha c z^{2} \sum_{i=1}^{k} r_{i}+\frac{1}{2} L \alpha c \sum_{i=1}^{k} \int_{t-r_{i}}^{t} y^{2}(s) d s, \\
& y c(t) \sum_{i=1}^{k} \int_{t-r_{i}}^{t} h_{i}^{\prime}(x(s)) y(s) d s \\
& \leq c(t)|y| \sum_{i=1}^{k} \int_{t-r_{i}}^{t} \rho_{i}|y(s)| d s \\
& \quad \leq \frac{1}{2} c(t) y^{2} \sum_{i=1}^{k} r_{i} \rho_{i}+\frac{1}{2} c(t) \sum_{i=1}^{k} \int_{t-r_{i}}^{t} \rho_{i} y^{2}(s) d s \\
& \quad \leq \frac{1}{2} L c y^{2} \sum_{i=1}^{k} r_{i}+\frac{1}{2} L c \sum_{i=1}^{k} \int_{t-r_{i}}^{t} y^{2}(s) d s,
\end{aligned}
$$

where

$$
c=\max \rho_{i} .
$$

Noting the previous discussion, it follows that

$$
\begin{aligned}
\frac{d}{d t} V(\cdot) \leq & c^{\prime}(t) H(x)+n \alpha b^{\prime}(t) G(y)+\alpha c^{\prime}(t) y \sum_{i=1}^{k} h_{i}(x) \\
& +\frac{1}{2} a^{\prime}(t) y^{2} \\
& -\left[n y b(t) g(y)-\alpha c(t) y^{2} \sum_{i=1}^{k} h_{i}^{\prime}(x)\right. \\
& \left.-\sum_{i=1}^{k} \lambda_{i} r_{i} y^{2}\right]
\end{aligned}
$$$$
-\frac{1}{2}\left[2(\alpha a(t)-1)-L \alpha c \sum_{i=1}^{k} r_{i}\right] z^{2}+\frac{1}{2} L c y^{2} \sum_{i=1}^{k} r_{i}
$$$$
+\sum_{i=1}^{k}\left[\frac{1}{2} L c(\alpha+1)-\lambda_{i}\right] \int_{t-r_{i}}^{t} y^{2}(s) d s
$$

If $y=0$, then

$$
n y b(t) g(y)-\alpha c(t) y^{2} \sum_{i=1}^{k} h_{i}^{\prime}(x)-\sum_{i=1}^{k} \lambda_{i} r_{i} y^{2}=0 .
$$

If $y \neq 0$, then it follows that

$$
\begin{gathered}
n y b(t) g(y)-\alpha c(t) y^{2} \sum_{i=1}^{k} h_{i}^{\prime}(x)-\sum_{i=1}^{k} \lambda_{i} r_{i} y^{2} \\
=\left[n b(t) \frac{g(y)}{y}-\alpha c(t) \sum_{i=1}^{k} h_{i}^{\prime}(x)-\sum_{i=1}^{k} \lambda_{i} r_{i}\right] y^{2} \\
\geq\left[n b(t) b-\alpha c(t) \sum_{i=1}^{k} \rho_{i}-\sum_{i=1}^{k} \lambda_{i} r_{i}\right] y^{2} \\
=c(t)\left[\frac{b(t)}{c(t)} n b-\alpha \sum_{i=1}^{k} \rho_{i}\right] y^{2}-\sum_{i=1}^{k} \lambda_{i} r_{i} y^{2} \\
\geq \delta_{1}\left[n b-\alpha \sum_{i=1}^{k} \rho_{i}-\delta_{1}^{-1} \sum_{i=1}^{k} \lambda_{i} r_{i}\right] y^{2} .
\end{gathered}
$$


Since $0 \leq \delta_{1} \leq c(t) \leq b(t), 1 \leq b(t) / c(t)$, and $\alpha a(t) \geq$ $\alpha a>1$, then

$$
\begin{aligned}
& \frac{1}{2} a^{\prime}(t) y^{2} \\
& \quad-\left[n y b(t) g(y)-\alpha c(t) y^{2} \sum_{i=1}^{k} h_{i}^{\prime}(x)-\sum_{i=1}^{k} \lambda_{i} r_{i} y^{2}\right] \\
& \leq\left[\delta_{2}-\delta_{1}\left(n b-\alpha \sum_{i=1}^{k} \rho_{i}\right)+\sum_{i=1}^{k} \lambda_{i} r_{i}\right] y^{2} \\
& =-\left(\delta_{5}-\sum_{i=1}^{k} \lambda_{i} r_{i}\right) y^{2} \\
& {\left[2(\alpha a(t)-1)-L \alpha c \sum_{i=1}^{k} r_{i}\right] z^{2} \geq\left(\delta_{6}-L \alpha c \sum_{i=1}^{k} r_{i}\right) z^{2},}
\end{aligned}
$$

where $\delta_{5}=\delta_{1}\left(n b-\alpha \sum_{i=1}^{k} \rho_{i}\right)-\delta_{2}>0$ and $\delta_{6} \equiv 2(\alpha a-1)>0$. Thus, we get

$$
\begin{aligned}
\frac{d}{d t} V(\cdot) \leq & c^{\prime}(t) H(x)+n \alpha b^{\prime}(t) G(y)+\alpha c^{\prime}(t) y \sum_{i=1}^{k} h_{i}(x) \\
& -\left\{\delta_{5}-\sum_{i=1}^{k} \lambda_{i} r_{i}\right\} y^{2}-\frac{1}{2}\left(\delta_{6}-L \alpha c \sum_{i=1}^{k} r_{i}\right) z^{2} \\
& +\frac{1}{2} L c y^{2} \sum_{i=1}^{k} r_{i} \\
+ & \sum_{i=1}^{k}\left[\frac{1}{2} L c(\alpha+1)-\lambda_{i}\right] \int_{t-r_{i}}^{t} y^{2}(s) d s .
\end{aligned}
$$

Let $\lambda_{i}=(1 / 2) L c(\alpha+1)$. Hence,

$$
\begin{aligned}
\frac{d}{d t} V(\cdot) & \\
\leq & c^{\prime}(t) H(x)+n \alpha b^{\prime}(t) G(y)+\alpha c^{\prime}(t) y \sum_{i=1}^{k} h_{i}(x) \\
& -\left\{\delta_{5}-\frac{1}{2} L c(\alpha+2) \sum_{i=1}^{k} r_{i}\right\} y^{2} \\
& -\frac{1}{2}\left(\delta_{6}-L \alpha c \sum_{i=1}^{k} r_{i}\right) z^{2} .
\end{aligned}
$$

If $c^{\prime}(t)=0$, then $c^{\prime}(t) H(x)+n \alpha b^{\prime}(t) G(y)+\alpha c^{\prime}(t) y$ $\sum_{i=1}^{k} h_{i}(x)=n \alpha b^{\prime}(t) G(y) \leq 0$ since $b^{\prime}(t) \leq 0$ and $G(y) \geq 0$. For those $t^{\prime}$ s such that $c^{\prime}(t)<0$, we have

$$
\begin{aligned}
c^{\prime}(t) H(x)+n \alpha b^{\prime}(t) G(y)+\alpha c^{\prime}(t) y \sum_{i=1}^{k} h_{i}(x) \\
=c^{\prime}(t)\left[H(x)+n \alpha \frac{b^{\prime}(t)}{c^{\prime}(t)} G(y)+\alpha y \sum_{i=1}^{k} h_{i}(x)\right] \\
\leq c^{\prime}(t)\left[H(x)+n \alpha G(y)+\alpha y \sum_{i=1}^{k} h_{i}(x)\right] \\
\leq c^{\prime}(t) \delta_{4} H(x) \leq 0 .
\end{aligned}
$$

Thus,

$$
\begin{aligned}
\frac{d}{d t} V(\cdot) \leq & -\left\{\delta_{5}-\frac{1}{2} L c(\alpha+2) \sum_{i=1}^{k} r_{i}\right\} y^{2} \\
& -\frac{1}{2}\left(\delta_{6}-L \alpha c \sum_{i=1}^{k} r_{i}\right) z^{2}
\end{aligned}
$$

Therefore, if

$$
\sum_{i=1}^{k} r_{i}<\min \left\{\frac{2 \delta_{5}}{L c(\alpha+2)}, \frac{\delta_{6}}{L \alpha c}\right\},
$$

then we have

$$
\frac{d}{d t} V(\cdot) \leq-\beta\left(y^{2}+z^{2}\right) \quad \text { for some } \beta>0 .
$$

The proof for Theorem 1 is complete.

Let $p(t) \neq 0$.

Theorem 3. One assumes that all the assumptions of Theorem 1 and the assumption

$$
\int_{0}^{t}|p(s)| d s<\infty
$$

hold. If

$$
\begin{gathered}
\frac{b}{\rho_{i}}>\alpha>\frac{1}{a}, \\
\frac{1}{2} a^{\prime}(t) \leq \delta_{2}<\delta_{1}\left(n b-\alpha \sum_{i=1}^{k} \rho_{i}\right), \\
\sum_{i=1}^{k} r_{i}<\min \left\{\frac{2 \delta_{5}}{L c(\alpha+2)}, \frac{\delta_{6}}{L c \alpha}\right\},
\end{gathered}
$$

then all solutions of (2) are bounded. 
Proof. Equation (2) is equivalent to the system

$$
\begin{gathered}
\dot{x}=y, \\
\dot{y}=z, \\
\dot{z}=-a(t) z-n b(t) g(y)-c(t) \sum_{i=1}^{k} h_{i}(x(t)) \\
+c(t) \sum_{i=1}^{k} \int_{t-r_{i}}^{t} h_{i}^{\prime}(x(s)) y(s) d s+p(t) .
\end{gathered}
$$

Along any solution $(x(t), y(t), z(t))$ of (6), we have

$$
\dot{V}_{(3)}(\cdot)=\dot{V}_{(2)}(\cdot)+(y+\alpha z) p(t) .
$$

Since $\dot{V}_{(2)}(\cdot) \leq 0$, then it follows that

$$
\begin{aligned}
\dot{V}_{(3)}(\cdot) & \leq(y+\alpha z) p(t) \leq(|y|+\alpha|z|)|p(t)| \\
& \leq \delta_{8}(|y|+|z|)|p(t)|,
\end{aligned}
$$

where $\delta_{8} \equiv \max \{1, \alpha\}$. Noting that $|m|<1+m^{2}$, we get

$$
\begin{aligned}
\dot{V}_{(3)}(\cdot) & \leq \delta_{8}\left(2+y^{2}+z^{2}\right)|p(t)| \leq 2 \delta_{8}|p(t)|+\delta_{8}\|X\|^{2}|p(t)| \\
& \leq 2 \delta_{8}|p(t)|+\left(\frac{\delta_{8}}{\delta_{7}}\right) V(\cdot)|p(t)|
\end{aligned}
$$

recalling that $\delta_{7}\|X\|^{2} \leq V(\cdot)$.

Let $\eta=\max \left(2 \delta_{8}, \delta_{8} / \delta_{7}\right)$, then

$$
\dot{V}_{(3)}(\cdot) \leq \eta|p(t)|+\eta V(\cdot)|p(t)|
$$

or

$$
\dot{V}_{(3)}(\cdot)-\eta V(\cdot)|p(t)| \leq \eta|p(t)| \text {. }
$$

Multiplying each side of this estimate by the integrating factor $\exp \left(-\eta \int_{0}^{t}|p(s)| d s\right)$, we get

$$
\begin{aligned}
\dot{V}_{(3)}(\cdot) & \exp \left(-\eta \int_{0}^{t}|p(s)| d s\right) \\
& -\eta V(\cdot)|p(t)| \exp \left(-\eta \int_{0}^{t}|p(s)| d s\right) \\
\leq & \eta|p(t)| \exp \left(-\eta \int_{0}^{t}|p(s)| d s\right) .
\end{aligned}
$$

Integrating each side of this estimate from 0 to $t$, we obtain

$$
\begin{array}{r}
V(\cdot) \exp \left(-\eta \int_{0}^{t}|p(s)| d s\right)-V(0) \\
\leq 1-\exp \left(-\eta \int_{0}^{t}|p(s)| d s\right)
\end{array}
$$

or

$$
V(\cdot) \leq V(0) \exp \left(\eta \int_{0}^{t}|p(s)| d s\right)+\exp \left(\eta \int_{0}^{t}|p(s)| d s\right)-1,
$$

where $(0, x(0), y(0), z(0))=0$.

Since $\int_{0}^{t}|p(s)| d s \leq A$ for all $t$, this implies

$$
V(\cdot) \leq V(0) e^{\eta A}+\left(e^{\eta A}-1\right) \quad \text { for } t \geq 0 .
$$

Since the right-hand side of the last estimate is a constant and $V(\cdot) \rightarrow \infty$ when $x^{2}+y^{2}+z^{2} \rightarrow \infty$, it follows that there exists a positive constant $D$ such that

$$
|x(t)| \leq D, \quad|y(t)| \leq D, \quad|z(t)| \leq D \quad \forall t \geq 0 .
$$

From the system (30) this implies that

$$
|x(t)| \leq D, \quad|\dot{x}(t)| \leq D, \quad|\ddot{x}(t)| \leq D \quad \forall t \geq 0 .
$$

The proof for Theorem 3 is complete.

\section{References}

[1] K. E. Chlouverakis and J. C. Sprott, "Chaotic hyperjerk systems," Chaos, Solitons \& Fractals, vol. 28, no. 3, pp. 739-746, 2006.

[2] J. Cronin-Scanlon, "Some mathematics of biological oscillations," SIAM Review, vol. 19, no. 1, pp. 100-138, 1977.

[3] R. Eichhorn, S. J. Linz, and P. Hänggi, “Transformations of nonlinear dynamical systems to jerky motion and its application to minimal chaotic flows," Physical Review E, vol. 58, no. 6, pp. 7151-7164, 1998.

[4] K. O. Friedrichs, "On nonlinear vibrations of third order," in Studies in Nonlinear Vibration Theory, pp. 65-103, Institute for Mathematics and Mechanics, New York University, 1946.

[5] S. J. Linz, "On hyperjerky systems," Chaos, Solitons \& Fractals, vol. 37, no. 3, pp. 741-747, 2008.

[6] L. L. Rauch, "Oscillation of a third order nonlinear autonomous system," in Contributions to the Theory of Nonlinear Oscillations, Annals of Mathematics Studies, no. 20, pp. 39-88, Princeton University Press, Princeton, NJ, USA, 1950.

[7] T. A. Ademola, M. O. Ogundiran, P. O. Arawomo, and O. A. Adesina, "Boundedness results for a certain third order nonlinear differential equation," Applied Mathematics and Computation, vol. 216, no. 10, pp. 3044-3049, 2010.

[8] A. U. Afuwape and J. E. Castellanos, "Asymptotic and exponential stability of certain third-order non-linear delayed differential equations: frequency domain method," Applied Mathematics and Computation, vol. 216, no. 3, pp. 940-950, 2010.

[9] E. N. Chukwu, "On the boundedness and the existence of a periodic solution of some nonlinear third order delay differential equation," Atti della Accademia Nazionale dei Lincei. Rendiconti. Classe di Scienze Fisiche, Matematiche e Naturali 8, vol. 64, no. 5, pp. 440-447, 1978.

[10] J. O. C. Ezeilo, "A stability result for a certain third order differential equation," Annali di Matematica Pura ed Applicata 4, vol. 72, pp. 1-9, 1966.

[11] J. O. C. Ezeilo and H. O. Tejumola, "Boundedness theorems for certain third order differential equations," Atti della Accademia Nazionale dei Lincei. Rendiconti. Classe di Scienze Fisiche, Matematiche e Naturali 8, vol. 55, pp. 194-201, 1973/1974. 
[12] T. Hara, "On the uniform ultimate boundedness of the solutions of certain third order differential equations," Journal of Mathematical Analysis and Applications, vol. 80, no. 2, pp. 533-544, 1981.

[13] B. Mehri and D. Shadman, "Boundedness of solutions of certain third order differential equation," Mathematical Inequalities \& Applications, vol. 2, no. 4, pp. 545-549, 1999.

[14] B. S. Ogundare and G. E. Okecha, "On the boundedness and the stability of solution to third order non-linear differential equations," Annals of Differential Equations, vol. 24, no. 1, pp. $1-8,2008$

[15] M. O. Omeike, "Stability and boundedness of solutions of some non-autonomous delay differential equation of the third order," Analele Ştiinţifice ale Universităţii Al. I. Cuza din Iaşi. Serie Nouă. Matematică, vol. 55, supplement 1, pp. 49-58, 2009.

[16] R. Reissig, G. Sansone, and R. Conti, Non-Linear Differential Equations of Higher Order, Noordhoff International, Leyden, The Netherlands, 1974.

[17] K. E. Swick, "Asymptotic behavior of the solutions of certain third order differential equations," SIAM Journal on Applied Mathematics, vol. 19, pp. 96-102, 1970.

[18] H. O. Tejumola, "On the boundedness and periodicity of solutions of certain third-order non-linear differential equations," Annali di Matematica Pura ed Applicata 4, vol. 83, pp. 195-212, 1969.

[19] H. O. Tejumola, "A note on the boundedness and the stability of solutions of certain third-order differential equations," Annali di Matematica Pura ed Applicata 4, vol. 92, pp. 65-75, 1972.

[20] C. Tunç, "Uniform ultimate boundedness of the solutions of third-order nonlinear differential equations," Kuwait Journal of Science \& Engineering, vol. 32, no. 1, pp. 39-48, 2005.

[21] C. Tunç, "Boundedness of solutions of a third-order nonlinear differential equation," Journal of Inequalities in Pure and Applied Mathematics, vol. 6, no. 1, article 3, 6 pages, 2005.

[22] C. Tunç, "On the asymptotic behavior of solutions of certain third-order nonlinear differential equations," Journal of Applied Mathematics and Stochastic Analysis, no. 1, pp. 29-35, 2005.

[23] C. Tunç, "Stability criteria for certain third order nonlinear delay differential equations," Portugaliae Mathematica, vol. 66, no. 1, pp. 71-80, 2009.

[24] C. Tunç, "A new result on the stability of solutions of a nonlinear differential equation of third-order with finite lag," Southeast Asian Bulletin of Mathematics, vol. 33, no. 5, pp. 947-958, 2009.

[25] C. Tunç, "On the stability and boundedness of solutions to third order nonlinear differential equations with retarded argument," Nonlinear Dynamics, vol. 57, no. 1-2, pp. 97-106, 2009.

[26] C. Tunç, "The boundedness of solutions to nonlinear third order differential equations," Nonlinear Dynamics and Systems Theory, vol. 10, no. 1, pp. 97-102, 2010.

[27] C. Tunç, "On the stability and boundedness of solutions of nonlinear third order differential equations with delay," Filomat, vol. 24, no. 3, pp. 1-10, 2010.

[28] C. Tunç, "Some stability and boundedness conditions for nonautonomous differential equations with deviating arguments," Electronic Journal of Qualitative Theory of Differential Equations, no. 1,12 pages, 2010.

[29] C. Tunç, "Bound of solutions to third-order nonlinear differential equations with bounded delay," Journal of the Franklin Institute, vol. 347, no. 2, pp. 415-425, 2010.

[30] C. Tunç, "Stability and bounded of solutions to nonautonomous delay differential equations of third order," Nonlinear Dynamics, vol. 62, no. 4, pp. 945-953, 2010.
[31] C. Tunç, "Existence of periodic solutions to nonlinear differential equations of third order with multiple deviating arguments," International Journal of Differential Equations, vol. 2012, Article ID 406835, 13 pages, 2012.

[32] C. Tunç, "Qualitative behaviors of functional differential equations of third order with multiple deviating arguments," Abstract and Applied Analysis, vol. 2012, Article ID 392386, 12 pages, 2012.

[33] C. Tunç, "On the qualitative behaviors of solutions of some differential equations of higher order with multiple deviating arguments," Journal of the Franklin Institute. In press.

[34] T. Yoshizawa, Stability Theory by Liapunov's Second Method, Publications of the Mathematical Society of Japan, No. 9, The Mathematical Society of Japan, Tokyo, Japan, 1966.

[35] N. N. Krasovskiì, Stability of Motion. Applications of Lyapunov's Second Method to Differential Systems and Equations with Delay, Stanford University Press, Stanford, Calif, USA, 1963. 


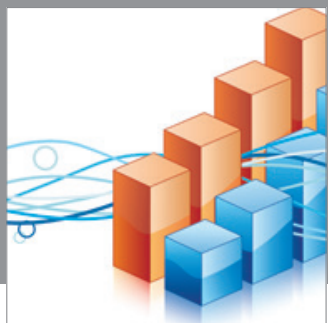

Advances in

Operations Research

mansans

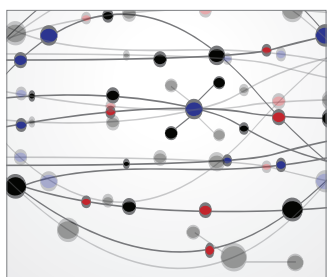

The Scientific World Journal
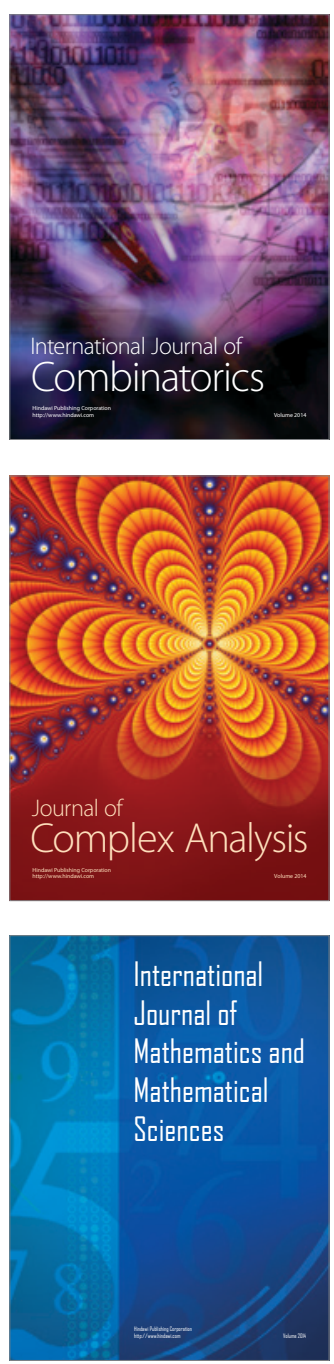
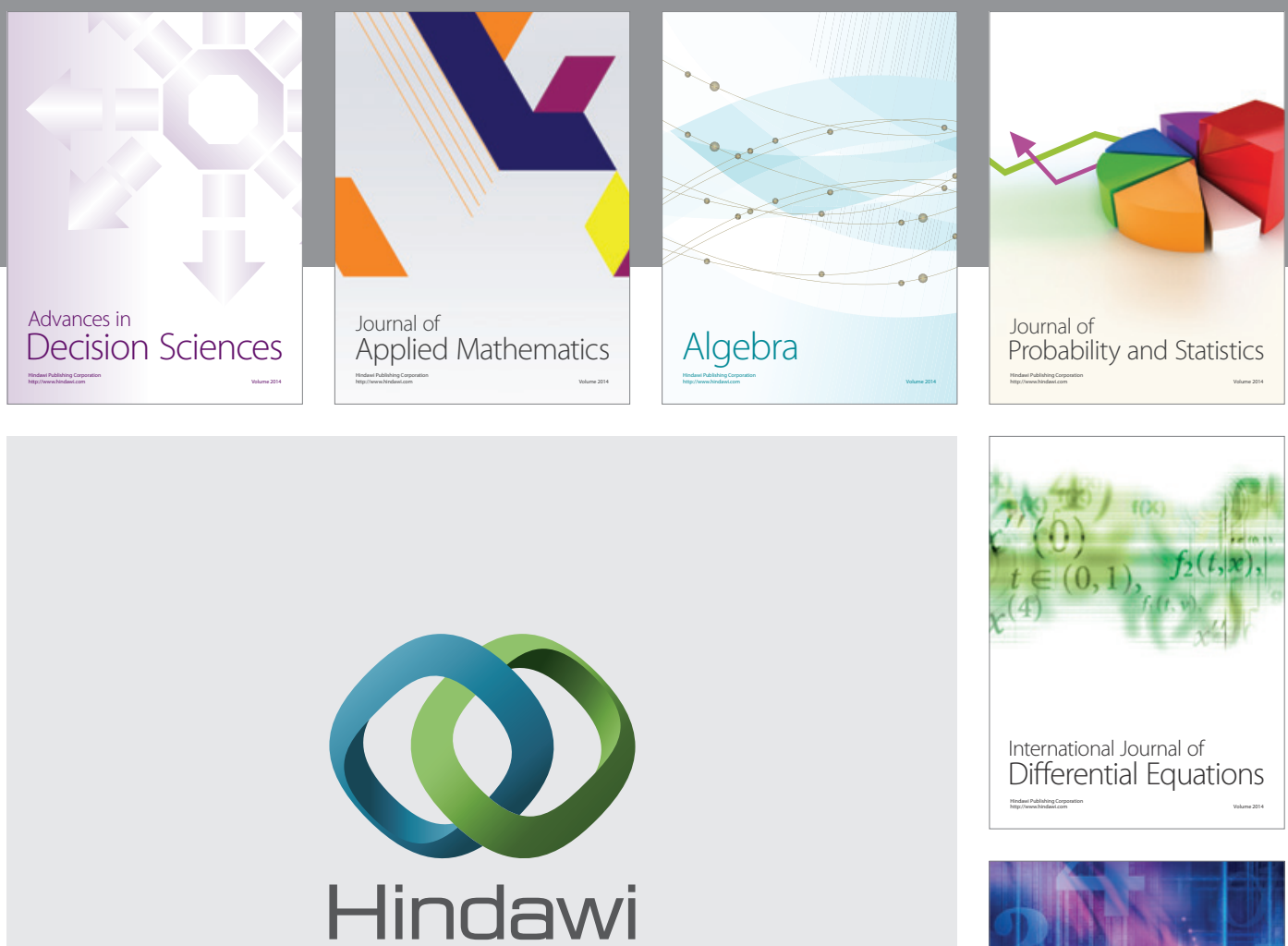

Submit your manuscripts at http://www.hindawi.com
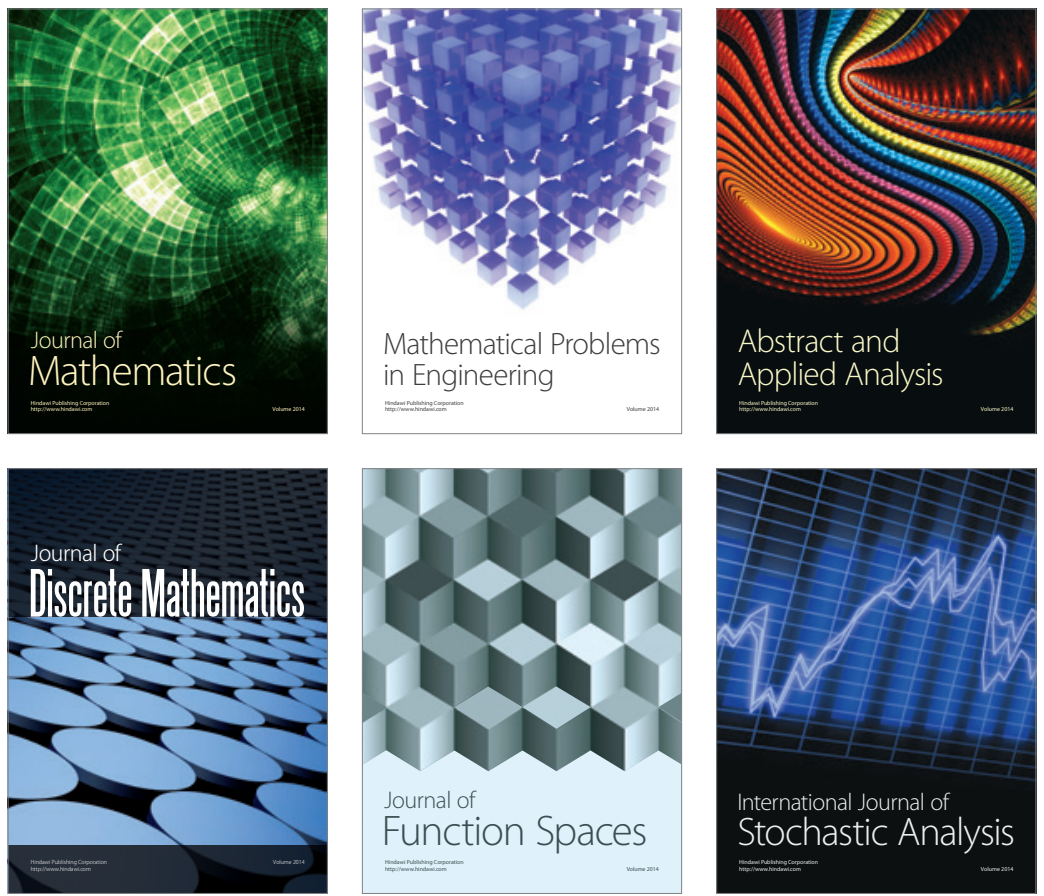

Journal of

Function Spaces

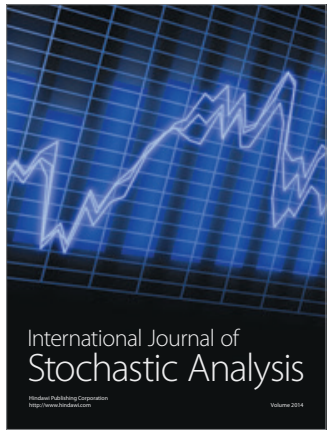

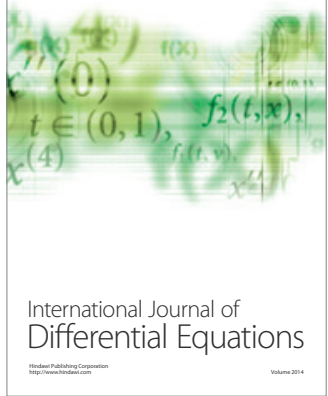
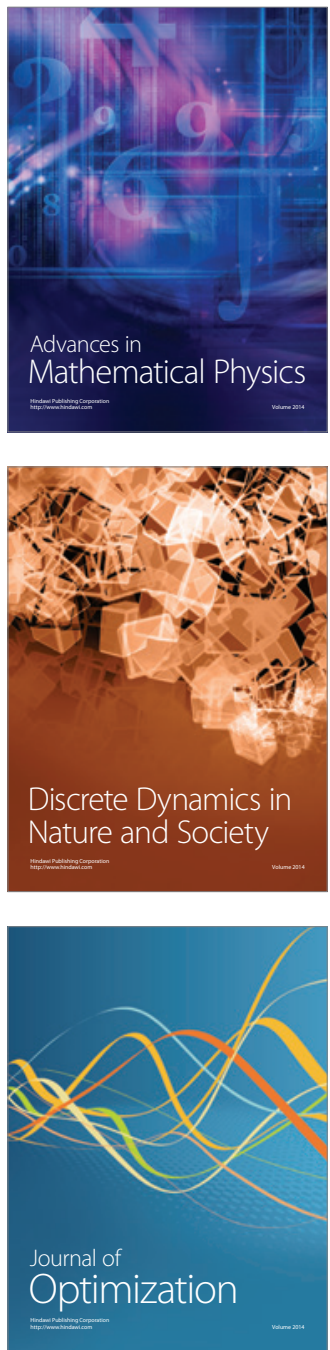